\title{
A NEW APPROACH IN OBTAINING A BETTER ESTIMATION IN APPROXIMATION BY POSITIVE LINEAR OPERATORS
}

\author{
M. ALI ÖZARSLAN AND OKTAY DUMAN
}

\begin{abstract}
In this study, without preserving some test functions, we present a new approach in obtaining a better error estimation in the approximation by means of positive linear operators. We also show that our method can be applied to many well-known approximation operators.
\end{abstract}

\section{INTRODUCTION}

Obtaining better error estimations in approximation to a function by a sequence of positive linear operators is an important problem in the approximation theory. So far, some relating results have been presented for Bernstein polynomials [5], Szász-Mirakjan operators [4], Meyer-König and Zeller operators [7] and BernsteinChlodovsky operators [1] by preserving some test functions in the approximation. Recently, Agratini [2] has applied a similar idea to more general summation-type positive linear operators. However, in this note, without preserving the test functions we introduce a different approach in order to get a faster approximation. We show that our new method can easily be applied to many well-known positive linear operators.

Let $\mathbb{R}^{+}:=[0, \infty)$ and $\mathbb{R}_{b}^{+}:=[0, b]$ with $b>0$. Consider the function space $E\left(\mathbb{R}^{+}\right)$ defined by

$$
E\left(\mathbb{R}^{+}\right):=\left\{f \in C\left(\mathbb{R}^{+}\right): \lim _{x \rightarrow \infty} \frac{f(x)}{1+x^{2}} \text { is finite }\right\}
$$

endowed with the norm

$$
\|f\|_{+}=\sup _{x \in \mathbb{R}^{+}} \frac{|f(x)|}{1+x^{2}} .
$$

However, for the bounded interval $\mathbb{R}_{b}^{+}$, we will consider the function space $C\left(\mathbb{R}_{b}^{+}\right)$ and the usual maximum norm $\|\cdot\|$ on $\mathbb{R}_{b}^{+}$.

Received by the editors March 16, 2009, Accepted: June. 05, 2009.

1991 Mathematics Subject Classification. 41A25, 41A36.

Key words and phrases. The Korovkin theorem, Bernstein polynomials, Szász-Mirakjan operators, Bernstein-Kantorovich operators, rate of convergence. 
Throughout the paper we use the test functions $f_{i}(x)=x^{i}$ for $i=0,1,2$. Assume that a sequence $\left\{L_{n}\right\}$ of positive linear operators defined on $E\left(\mathbb{R}^{+}\right)$(or, $C\left(\mathbb{R}_{b}^{+}\right)$) satisfies the following conditions:

$$
L_{n}\left(f_{0} ; x\right)=1, L_{n}\left(f_{1} ; x\right)=a_{n} x+b_{n}, L_{n}\left(f_{2} ; x\right)=c_{n} x^{2}+d_{n} x+e_{n},
$$

where $\left(a_{n}\right),\left(b_{n}\right),\left(c_{n}\right),\left(d_{n}\right)$ and $\left(e_{n}\right)$ are sequences of non-negative real numbers satisfying the following conditions:

$$
\lim _{n \rightarrow \infty} a_{n}=\lim _{n \rightarrow \infty} c_{n}=1\left(c_{n} \neq 0\right), \quad \lim _{n \rightarrow \infty} b_{n}=\lim _{n \rightarrow \infty} d_{n}=\lim _{n \rightarrow \infty} e_{n}=0 .
$$

Actually, many well-known approximation operators, such as Bernstein polynomials, Szász-Mirakjan operators, Bernstein-Kantorovich operators etc., satisfy the conditions (1.1) and (1.2). Our primary interest of this paper is to construct positive linear operators providing better error estimates than the operators $L_{n}$ as given above.

Now consider the lattice homomorphism $T_{b}: C\left(\mathbb{R}^{+}\right) \rightarrow C\left(\mathbb{R}_{b}^{+}\right)$defined by $T_{b}(f):=\left.f\right|_{\mathbb{R}_{b}^{+}}$for every $f \in C\left(\mathbb{R}^{+}\right)$. In this case, we see from the classical Korovkin theorem (see $[6, \mathrm{p} .14])$ that

$$
\lim _{n \rightarrow \infty} T_{b}\left(L_{n}(f)\right)=T_{b}(f) \text { uniformly on } \mathbb{R}_{b}^{+} \text {. }
$$

On the other hand, with the universal Korovkin-type property with respect to monotone operators (see Theorem 4.1.4 (vi) of [3, p. 199]) we have the following: "Let $X$ be a compact set and $H$ be a cofinal subspace of $C(X)$. If $E$ is a Banach lattice, $S: C(X) \rightarrow E$ is a lattice homomorphism and if $\left\{L_{n}\right\}$ is a sequence of positive linear operators from $C(X)$ into $E$ such that $\lim _{n \rightarrow \infty} L_{n}(h)=S(h)$ for all $h \in H$, then $\lim _{n \rightarrow \infty} L_{n}(f)=f$ provided that $f$ belongs to the Korovkin closure of $H^{\prime \prime}$.

Hence, by using (1.3) and the above property we obtain the following result.

Theorem 1.1. Let $\left\{L_{n}\right\}$ be a sequence of positive linear operators defined on $E\left(\mathbb{R}^{+}\right)$ (resp. $C\left(\mathbb{R}_{b}^{+}\right)$) satisfying the conditions in (1.1) and (1.2). Then, for all $f \in E\left(\mathbb{R}^{+}\right)$ (resp. for all $f \in C\left(\mathbb{R}_{b}^{+}\right)$), we have $\lim _{n \rightarrow \infty} L_{n}(f)=f$ uniformly on the interval $\mathbb{R}_{b}^{+}$with $b>0$.

\section{Better Error Estimates}

Let $A$ denote $\mathbb{R}^{+}$or $\mathbb{R}_{b}^{+}$. For each $x \in A$, consider the first central moment function $\psi_{x}$ defined by $\psi_{x}(y)=y-x$. Assume that $I$ be a subinterval of $A$. Now in order to get a better error estimation in the approximation by means of the operators $L_{n}$ (cf. Theorem 1.1) we look for a functional sequence $\left(u_{n}\right), u_{n}: I \rightarrow A$, such that

$$
\delta_{n}^{*}(x):=\sqrt{L_{n}\left(\psi_{x}^{2} ; u_{n}(x)\right)} \leq \sqrt{L_{n}\left(\psi_{x}^{2} ; x\right)}=: \delta_{n}(x) \text { for } x \in I .
$$

By (1.1), this is equivalent to

$$
c_{n} u_{n}^{2}(x)+\left(d_{n}-2 a_{n} x\right) u_{n}(x)-\left(c_{n}-2 a_{n}\right) x^{2}-d_{n} x \leq 0 .
$$


Now let

$$
\Delta_{n}(x):=\left(d_{n}-2 a_{n} x\right)^{2}+4 c_{n}\left\{\left(c_{n}-2 a_{n}\right) x^{2}+d_{n} x\right\} .
$$

Assume that there exist a subinterval $I \subset A$ and a number $n_{0} \in \mathbb{N}$ such that

$$
\Delta_{n}(x) \geq 0
$$

and

$$
\frac{2 a_{n} x-d_{n}}{2 c_{n}} \in A
$$

hold for every $x \in I$ and for every $n \geq n_{0}$. In this case, from (2.2), (2.3) and (2.4), we get

$$
s_{n}(x):=\frac{2 a_{n} x-d_{n}-\sqrt{\Delta_{n}(x)}}{2 c_{n}} \leq u_{n}(x) \leq \frac{2 a_{n} x-d_{n}+\sqrt{\Delta_{n}(x)}}{2 c_{n}}=: t_{n}(x) .
$$

Hence, we can choose, e.g.,

$$
u_{n}(x):=\frac{s_{n}(x)+t_{n}(x)}{2}=\frac{2 a_{n} x-d_{n}}{2 c_{n}} .
$$

In this case, we can define a new positive linear operator as follows:

$$
L_{n}^{*}(f ; x):=L_{n}\left(f ; u_{n}(x)\right), \quad x \in I .
$$

It is well known that if a positive linear operator $U$ defined on $C_{B}(K)$, the space of all continuous bounded functions on an interval $K \subset \mathbb{R}$, preserves the test function $f_{0}$, then it satisfies

$$
|U(f ; x)-f(x)| \leq 2 \omega\left(f, \sqrt{U\left(\psi_{x}^{2} ; x\right)}\right),
$$

where $\omega(f, \delta), \delta>0$, denotes the modulus of continuity of a continuous (and bounded) function $f$ on $K$.

Now let $A=\mathbb{R}^{+}$(or $\mathbb{R}_{b}^{+}$) as stated before. Then, the last inequality implies that

$$
\left|L_{n}^{*}(f ; x)-f(x)\right| \leq 2 \omega\left(f, \delta_{n}^{*}(x)\right), \quad x \in I \subseteq A,
$$

and

$$
\left|L_{n}(f ; x)-f(x)\right| \leq 2 \omega\left(f, \delta_{n}(x)\right), \quad x \in A .
$$

Therefore, this means that the error estimation in the approximation by the modified operators $L_{n}^{*}$ is better than the approximation by the original operators $L_{n}$.

\section{Applications}

3.1. Bernstein Polynomials. Take $A=[0,1]$ and consider the classical Bernstein polynomials

$$
B_{n}(f ; x)=\sum_{k=0}^{n}\left(\begin{array}{l}
n \\
k
\end{array}\right) f\left(\frac{k}{n}\right) x^{k}(1-x)^{n-k},
$$


where $f \in C[0,1], n \in \mathbb{N}$ and $x \in[0,1]$. Since

$$
B_{n}\left(f_{0} ; x\right)=1, B_{n}\left(f_{1} ; x\right)=x, B_{n}\left(f_{2} ; x\right)=\left(1-\frac{1}{n}\right) x^{2}+\frac{1}{n} x,
$$

we take

$$
a_{n}=1, b_{n}=e_{n}=0, c_{n}=1-\frac{1}{n}, d_{n}=\frac{1}{n}
$$

for all $n \in \mathbb{N}$. Now observe that

$$
u_{n}(x)=\frac{2 a_{n} x-d_{n}}{2 c_{n}}=\frac{2 n x-1}{2(n-1)} \in[0,1]
$$

if and only if

$$
\frac{1}{2 n} \leq x \leq 1-\frac{1}{2 n} \text { for } n \geq 2
$$

So, choosing

$$
I=\left[\frac{1}{4}, \frac{3}{4}\right] \subset[0,1]
$$

we can easily show that if $x \in I$ and $n \geq 2$, then $u_{n}(x) \in[0,1]$. Furthermore, the choices in (3.2) are the best possible with respect to the functions $u_{n}^{*}(x)$ in (3.1). With these choices, our new operators are defined as follows:

$$
B_{n}^{*}(f ; x)=\frac{1}{2^{n}(n-1)^{n}} \sum_{k=0}^{n}\left(\begin{array}{l}
n \\
k
\end{array}\right) f\left(\frac{k}{n}\right)(2 n x-1)^{k}(2 n-2 n x-1)^{n-k},
$$

where $f \in C[0,1], x \in\left[\frac{1}{4}, \frac{3}{4}\right]$ and $n \geq 2$. According to this application, observe that

$$
\delta_{n}(x)=\sqrt{B_{n}\left(\psi_{x}^{2} ; x\right)}=\sqrt{\frac{x(1-x)}{n}}, \quad x \in[0,1] \text { and } n \geq 1,
$$

and

$$
\delta_{n}^{*}(x)=\sqrt{B_{n}^{*}\left(\psi_{x}^{2} ; x\right)}=\frac{1}{2} \sqrt{\frac{4 n x-4 n x^{2}-1}{n(n-1)}}, \quad x \in\left[\frac{1}{4}, \frac{3}{4}\right] \text { and } n \geq 2 .
$$

Then one can say that the convergence to zero, as $n \rightarrow \infty$, of the sequence $\left\{B_{n}^{*}(f ; x)-f(x)\right\}$ is faster than that of $\left\{B_{n}(f ; x)-f(x)\right\}$ on the best possible interval $\left[\frac{1}{4}, \frac{3}{4}\right]$ with respect to the functions $u_{n}(x)$ given by $(3.1)$.

We know that the values $\delta_{n}(x)$ and $\delta_{n}^{*}(x)$ actually control the rate of the approximation a function by means of $B_{n}$ and $B_{n}^{*}$, respectively. Therefore, one can say that the error estimation in the approximation by $B_{n}^{*}$ is more sensitive than by the classical Bernstein polynomials $B_{n}$. 
3.2. Szász-Mirakjan Operators. Take $A=\mathbb{R}^{+}$and consider the classical SzászMirakjan operators

$$
S_{n}(f ; x)=e^{-n x} \sum_{k=0}^{\infty} f\left(\frac{k}{n}\right) \frac{(n x)^{k}}{k !},
$$

where $f \in E\left(\mathbb{R}^{+}\right), n \in \mathbb{N}$ and $x \in \mathbb{R}^{+}$. Then, by a simple calculation, we get

$$
u_{n}(x)=x-\frac{1}{2 n} \text {. }
$$

So, $u_{n}(x) \in \mathbb{R}^{+}$if and only if $x \geq \frac{1}{2 n}$ and $n \geq 1$. Hence, choosing

$$
I=\left[\frac{1}{2}, \infty\right) \subset \mathbb{R}^{+},
$$

if $x \in I$ and $n \geq 1$, then we have $u_{n}^{*}(x) \in \mathbb{R}^{+}$. So, our modified operators are defined as follows:

$$
S_{n}^{*}(f ; x)=e^{-n x+\frac{1}{2}} \sum_{k=0}^{\infty} f\left(\frac{k}{n}\right) \frac{(2 n x-1)^{k}}{2^{k} k !},
$$

where $f \in E\left(\mathbb{R}^{+}\right), x \in\left[\frac{1}{2}, \infty\right)$ and $n \in \mathbb{N}$. Then, one can say that, for all $f \in E\left(\mathbb{R}^{+}\right)$, the convergence to zero, as $n \rightarrow \infty$, of the sequence $\left\{S_{n}^{*}(f ; x)-f(x)\right\}$ is faster than that of $\left\{S_{n}(f ; x)-f(x)\right\}$ on the best possible interval $\left[\frac{1}{2}, \infty\right)$ with respect to the functions $u_{n}(x)$ given by (3.3).

3.3. Bernstein-Kantorovich Operators. Consider the classical

Bernstein-Kantorovich operators defined by

$$
U_{n}(f ; x):=(n+1) \sum_{k=0}^{n}\left(\begin{array}{l}
n \\
k
\end{array}\right) x^{k}(1-x)^{n-k} \int_{k /(n+1)}^{(k+1) /(n+1)} f(t) d t,
$$

where $f \in C[0,1], x \in A=[0,1]$ and $n \in \mathbb{N}$. In this case, we have

$$
u_{n}(x)=\frac{(n+1) x-1}{n-1} \in[0,1]
$$

if and only if

Hence, choosing

$$
\frac{1}{n+1} \leq x \leq \frac{n}{n+1} \text { and } n \geq 2
$$

$$
I=\left[\frac{1}{3}, \frac{2}{3}\right] \subset[0,1]
$$

we can easily show that if $x \in I$ and $n \geq 2$, then $u_{n}(x) \in[0,1]$. With these choices, our new operators are defined as follows:

$U_{n}^{*}(f ; x)=\frac{(n+1)}{(n-1)^{n}} \sum_{k=0}^{n}\left(\begin{array}{l}n \\ k\end{array}\right)\{(n+1) x-1\}^{k}\{n-(n+1) x\}^{n-k} \int_{k /(n+1)}^{(k+1) /(n+1)} f(t) d t$, 
where $f \in C[0,1], x \in\left[\frac{1}{3}, \frac{2}{3}\right]$ and $n \geq 2$. Then, we conclude that, for all $f \in C[0,1]$, the convergence to zero, as $n \rightarrow \infty$, of the sequence $\left\{U_{n}^{*}(f ; x)-f(x)\right\}$ is faster than that of $\left\{U_{n}(f ; x)-f(x)\right\}$ on the best possible interval $\left[\frac{1}{3}, \frac{2}{3}\right]$ with respect to the functions $u_{n}(x)$ given by $(3.4)$.

Remark 3.1. Our new approach can also be applied to other well-known approximation operators. But, we omit the details.

ÖZET: Bu çalışmada, pozitif doğrusal operatörlerle yaklaşımda daha iyi hata tahminleri elde edebilmek için bazı test fonksiyonların operatörler tarafından korunmasına ihtiyaç duyulmaksızın yeni bir yaklaşım metodu sunulmaktadır. Ayrıca buradaki metodun bilinen pek çok yaklaşım operatörlerine de uygulanabildiği gösterilmektedir.

\section{REFERENCES}

[1] O. Agratini, Linear operators that preserve some test functions. Int. J. Math. Math. Sci. Art.ID 94136 (2006), 11 pp.

[2] O. Agratini, On the iterates of a class of summation-type linear positive operators, Comput. Math. Appl. 55 (2008) 1178-1180.

[3] F. Altomare and M. Campiti, Korovkin-type Approximation Theory and its Application, Walter de Gruyter Studies in Math. 17, de Gruyter \& Co., Berlin, 1994.

[4] O. Duman and M.A. Özarslan, Szász-Mirakjan type operators providing a better error estimation, Appl. Math. Lett. 20 (2007) 1184-1188.

[5] J.P. King, Positive linear operators which preserve $x^{2}$, Acta. Math. Hungar. 99 (2003) 203-208.

[6] P.P. Korovkin, Linear Operators and Approximation Theory, Hindustan Publ. Co., Delhi, India, 1960.

[7] M.A. Özarslan and O. Duman, MKZ type operators providing a better estimation on $[1 / 2,1)$, Canadian Math. Bull. 50 (2007) 434-439.

Current address: M. Ali Özarslan: Eastern Mediterranean University, Faculty of Arts and Sciences, Department of Mathematics, Gazimagusa, Mersin 10, Turkey ,

Oktay Duman: TOBB Economics and Technology University, Faculty of Arts and Sciences, Department of Mathematics, Söğütözü 06530, Ankara, Turkey

E-mail address: mehmetali.ozarslan@emu.edu.tr, oduman@etu.edu.tr 